TE CHN IS C HE U N I VERS ITÄT B ERLIN

$$
\text { S'5371 }
$$

\title{
GENERATING HIGHER ORDER CODAZZI TENSORS
}

BY FUNCTIONS

Judith LEDER

Angela SCHWENK-SCHELLSCHMIDT

Udo SIMON

Martin WIEHE

Preprint No. 604/1998

P R E P R I N T R E I H E M A T H E M A T I K

FACHBEREICH 3 



\title{
Generating higher order Codazzi tensors by functions
}

\author{
J. Leder* \\ A. Schwenk-Schellschmidt** \\ U. Simon ${ }^{* *} \dagger$ \\ M. Wiehe*
}

Dedicated to the memory of Robert B. Gardner, UNC Chapel Hill

\begin{abstract}
We study higher order Codazzi tensors on constant curvature spaces and show how they can be generated by functions. We give applications in Riemannian geometry and hypersurface theory; in particular we characterize ellipsoids in terms of second order spherical harmonics.
\end{abstract}

Keywords: Codazzi tensors, spherical harmonics, Riemannian sphere, projective structures, quadrics. MS-Classification: 58G30, 33C35, 53A05, 53A15, 53C21, 53C40.

\section{Second order Codazzi tensors and first spherical harmonics}

The relations between $(0,2)$-Codazzi tensors on a Riemannian sphere $(M, g)$ of curvature $k>0$ and generating functions are well known. If $f \in C^{\infty}(M)$, then the symmetric $(0,2)$-tensor

$$
H(f):=\operatorname{Hess}(f)+k g f
$$

is a Codazzi tensor relative to the metric; here $\operatorname{Hess}(f)$ denotes the covariant Hessian of $f$. Moreover, any Codazzi tensor on $S^{n}(k)$ can be generated in this way and the generating function is unique modulo a first order spherical harmonic, as

$$
H(F)=0
$$

if and only if $F$ is a first order spherical harmonic.

This relation plays an important role for many global existence and uniqueness results in the theory of submanifolds, in particular when the third fundamental form III of a hypersurface is used as a metric of constant curvature $k=1$ on the spherical image. Well known examples are the Christoffel and Minkowski problem for ovaloids in Euclidean space, based on the fact that the second fundamental form II can be generated by the support function $\rho$ as in (1.0.1):

$$
\text { II }=\operatorname{Hess}_{\text {III }} \rho+\text { III } \rho \text {. }
$$

This relation is also the basis for the Monge-Ampère equation which was used for the solution of the Minkowski existence problem; [CY86], [Pog78]. In [OS83] global existence and uniqueness

\footnotetext{
*Supported by DFG (Graduiertenkolleg HU-TU Berlin)

** Partially supported by DFG

${ }^{\dagger}$ Partially supported by the exchange program TU Berlin - Emory U; it is a pleasure to thank V. Oliker for helpful discussions on the topic.
} 
problems for Codazzi tensors on arbitrary constant curvature spaces were investigated, based on the fact that one can generate Codazzi tensors by functions.

As far as we know it was Norden who first stated that, more generally, Codazzi tensors on projectively flat spaces can locally be generated by functions, see [SS62], p. 232; this includes the result for constant curvature spaces from [Fer91]. Norden's observation was systematically applied to solve certain PDEs on projectively flat spaces. These PDEs arise in particular in affine differential geometry; [PSS94], [Sim95], [GKS95]. The investigations recently were extended to Weyl geometries [BGS97].

Equation (1.0.2) appears in a more general context in Obata's theorem which characterizes Riemannian spheres [Oba62]. Tanno [Tan78] proved a similar result based on the third order PDE system characterizing second order spherical harmonics on the sphere which we state in standard local notation:

$$
\nabla_{k} \nabla_{j} f_{i}+k\left(2 f_{k} g_{i j}+f_{i} g_{j k}+f_{j} g_{k i}\right)=0 .
$$

We will take this equation as motivation to generate so called higher order Codazzi tensors by functions. An $(0, m)$-Codazzi tensor on a Riemannian manifold $(\mathrm{M}, \mathrm{g})$ is a symmetric $(0, m)$-tensor with totally symmetric covariant derivative. In $\S 2$ we summarize recent results on $(0, m)$-Codazzi tensors which we will apply in particular for $m=3$ and $m=4$.

In $\S 3-4$ we prove global existence and uniqueness results: we show how to generate $(0, m)$ Codazzi tensors on Riemannian spheres by functions for $m=3,4$. These global results are analogues of what is known for $m=2$ [OS83]. First applications in $\S 5$ are certain extensions of the results of Obata [Oba62] and Tanno [Tan78]. In $\S 6$ we state a general transformation lemma which describes the behaviour of Codazzi tensors under a projective change of a connection. $\S 7$ recalls definitions and elementary facts from relative hypersurface theory as a basis for applications in $\S 8$ of the results from $\S 3$ to Euclidean and affine hypersurface theory.

In $\S 8$ we derive a nonlinear third order PDE-system (in terms of the Levi-Civita connection of the third fundamental form) for the Euclidean support function of a non-degenerate hypersurface (i.e. its Gauß mapping is locally injective). We apply the results from $\S 3$ to this PDE-system and get local characterizations of proper affine spheres and quadrics. A particular corollary is the following global characterization of ellipsoids in terms of the Gauß-Kronecker curvature $H_{n}$ via spherical harmonics of the third fundamental form metric.

Theorem A. Let $x: M \rightarrow E$ be a hyperovaloid. Then $f$ is an hyperellipsoid if and only if the Gauß-Kronecker curvature $H_{n}$ satisfies

$$
\triangle^{*}\left(H_{n}^{\frac{2}{n+2}}-c\right)+2(n+1)\left(H_{n}^{\frac{2}{n+2}}-c\right)=0
$$

where $\triangle^{*}$ is the Laplacian of the third fundamental form metric $g^{*}=\mathrm{III}$ and $\omega^{*}$ its Riemannian volume form. The constant $c$ satisfies

$$
c=\frac{\int_{M} H_{n}^{\frac{2}{n+2}} \omega^{*}}{\int_{M} \omega^{*}} .
$$

Moreover, if $x$ is a hyperellipsoid given by $\sum\left(a_{i}\right)^{-2}\left(x^{i}\right)^{2}=1$ then

$$
c=\frac{\sum_{i=1}^{n+1} a_{i}^{2}}{(n+1)\left(a_{1} \cdots a_{n+1}\right)^{\frac{4}{n+2}}} .
$$

Theorem A will be proved at the end of $\S 8$. 


\section{Higher order Codazzi tensors}

In [LSW] higher order Codazzi tensors were introduced and investigated. We recall the definition and some of the results we are going to apply.

We use the following notation. Let $M$ be a connected, oriented, $n$-dimensional $C^{\infty}$-manifold, $n \geq 2$ and $u, v, w, \ldots$ vector fields on $M . \nabla$ denotes a torsion free affine connection, and $g$ a Riemannian metric with Riemannian volume form $\omega(g)$. In the case of a Riemannian manifold $(M, g)$ the connnection $\nabla$ is the Levi-Civita connection of $g$. The Laplace operator on $(M, g)$ is defined by

$$
\triangle f:=\operatorname{trace}_{g} \operatorname{Hess} f
$$

where Hess $f$ denotes the covariant Hessian of $f$ on $(M, g)$.

We adopt the sign of the Riemannian curvature tensor $R$ from [KN69], denote by Ric its Ricci tensor and by $k$ the normed scalar curvature $n(n-1) k=$ trace $_{g}$ Ric. If $M$ is a topological sphere with the standard differentiable structure, the notation is $M \cong S^{n}$. If moreover $(M, g)$ is isometric diffeomorphic to $\left(S^{n}\right.$, can $)$ where can is the canonical metric on $S^{n}$, the notation is $(M, g) \cong\left(S^{n}\right.$, can $)$.

\subsection{Definition.}

(i) Let $m \geq 1$. A totally symmetric tensor field $\Phi$ of order $(0, m)$ on $(M, \nabla)$ is called a $(0, m)$ Codazzi tensor relative to $\nabla$ if the covariant derivative $\nabla \Phi$ is totally symmetric; we call $\Phi$ also a $\nabla$-Codazzi tensor of order $m$. If $\nabla=\nabla(g)$ for some metric $g$, then we call $\Phi$ a Codazzi tensor on $(M, g)$.

(ii) We call $\Phi$ in (i) traceless with respect to the metric $g$ if $\operatorname{trace}_{g} \Phi=0$.

Examples of Codazzi tensors are well known for $m=2$, [LSW] contains a series of examples of Codazzi tensors of higher order $m \geq 2$. We are going to use results from this paper, which we summarize below.

2.2. Remark. If $\Phi$ is a $(0,1)$-Codazzi tensor relative to $\nabla$ then $\Phi$ locally can be generated by a function $\psi$, namely $\Phi=d \psi$, as the symmetry of $\nabla \Phi$ just is the integrability condition.

2.3. Theorem [LSW98]. Let $(M, \nabla)$ be a closed (that means compact without boundary) 2dimensional manifold of genus zero. Assume that $\Phi$ is a traceless $(0, m)$-Codazzi tensor with respect to some Riemannian metric $g$. Then $\Phi \equiv 0$.

2.4. Theorem [LSW]. Let $(M, g)$ be a closed Riemannian manifold of dimension $n>2$ and assume that the metric is conformally flat. Let $\Phi$ be a traceless $(0, m)$-Codazzi tensor. If the Ricci tensor is pinched

$$
\operatorname{Ric}>\frac{(m-1) n}{n+2 m-4} k g
$$

then $\Phi \equiv 0$.

2.5. Remark. Let $(M, g)$ be closed.

(i) If $(M, g)$ has constant positive curvature, then the assumptions in 2.4 are satisfied.

(ii) Assume that $(M, g)$ satisfies the conditions of 2.3 or 2.4 , resp. Let $\Phi, \Phi^{\#}$ be Codazzi tensors of order $m$ such that $\operatorname{trace}_{g} \Phi=\operatorname{trace}_{g} \Phi^{\#}$. Then $\Phi=\Phi^{\#}$. 
(iii) Let $\Phi$ be a Codazzi tensor of order $m$ and $1 \leq s \leq \frac{m}{2}, s \in \mathbb{N}$. Define the trace of order $s$ by

$$
\operatorname{trace}_{g}^{s} \Phi:=\operatorname{trace}_{g}\left(\operatorname{trace}_{g}^{s-1} \Phi\right), \quad \operatorname{trace}_{g}^{0} \Phi:=\Phi .
$$

Let $(M, g)$ be as in 2.3 or 2.4 , resp., and assume that the $(0, m)$-Codazzi tensors $\Phi, \Phi^{\#}$ satisfy $\operatorname{trace}_{g}^{s} \Phi=\operatorname{trace}_{g}^{s} \Phi^{\#}$. Then $\Phi=\Phi^{\#}$.

\section{Third order Codazzi tensors}

Let $(M, g)$ be a Riemannian space of constant curvature. Tanno's equation (1.0.4) gives the motivation how to generate third order Codazzi tensors on constant curvature spaces.

3.1. Lemma and Definition. Let $(M, g)$ be a space of constant curvature $k$.

(i) For $f \in C^{\infty}(M)$, the $(0,3)$-tensor $\Phi(f)$, defined in local terms by

$$
\begin{aligned}
\Phi(f)_{i j k} & :=\nabla_{k}\left(\nabla_{j} f_{i}+k f g_{i j}\right)+k\left\{f_{k} g_{i j}+f_{i} g_{j k}+f_{j} g_{k i}\right\} \\
& =\nabla_{k} H(f)_{i j}+k\{\cdots\} .
\end{aligned}
$$

is a Codazzi tensor on $(M, g)$. We say that $f$ generates $\Phi$.

(ii) Let $f, f^{\#}$ generate the same Codazzi tensor $\Phi(f)=\Phi=\Phi^{\#}(f)$. Then $f-f^{\#}=$ : F satisfies Tanno's equation (1.0.4) on $(M, g)$ :

$$
\Phi(F)=0
$$

(iii) We have $\operatorname{trace}_{g} \Phi(f)=d(\triangle f+2(n+1) k f)$.

Proof. (i) From (1.0.1) we know that $\nabla H(f)$ is totally symmetric while the expression $\{\cdots\}$ in (3.1.1) is obviously symmetric by definition. Thus $\Phi(f)$ is totally symmetric. The symmetry of $\nabla \Phi(f)$ follows by a straightforward calculation using the Ricci-identity for $(0,3)$-tensors (see e.g. [Eis49], p. 30).

(ii) is a trivial consequence of the linearity of the equation.

(iii) is obvious.

3.2. Notation. On $(M, g)$ denote by

$$
\begin{aligned}
& \mathfrak{C}_{0}:=\left\{f \in C^{\infty}(M) \mid f=\text { const }\right\}, \\
& \mathfrak{C}_{2}:=\left\{f \in C^{\infty}(M) \mid \Phi(f)=0 \text { on } M, f \notin \mathfrak{C}_{0}\right\} \cup\{0\},
\end{aligned}
$$

where $\Phi(f)$ was defined in (3.1.1). Obviously $\mathfrak{C}_{0}, \mathfrak{C}_{2}$ are $\mathbb{R}$-vector spaces, which on $S^{n}(k)$ are just eigenspaces of the Laplacian: $f \in \mathfrak{C}_{0}$ corresponds to the eigenvalue $\lambda=0$, while $f \in \mathfrak{C}_{2}$ is a second order spherical harmonic and $\lambda=2(n+1) k$. 
3.3. Definition and Lemma. Let $\Phi$ be a cubic form on a Riemannian manifold $(M, g)$.

(i) Then the equation

$$
n \widehat{T}=\operatorname{trace}_{g} \Phi
$$

defines the Tchebychev form $\widehat{T}$ of $\Phi$, while the Tchebychev field $T$ implicitly is defined by $g(T, v):=\widehat{T}(v)$.

(ii) If $\Phi$ is a $(0,3)$-Codazzi tensor on $(M, g)$ then $\widehat{T}=\frac{1}{n}$ trace $_{g} \Phi$ is a $(0,1)$-Codazzi tensor on $(M, g)$. In particular, $T$ is closed and $T$ locally can be generated by a function $\psi, d \psi=T$, and $\psi$ is unique modulo an additive constant.

3.4. Theorem. Let $(M, g)$ be isometrically diffeomorphic to $\left(S^{n}(k)\right.$, can $)$. Let $\bar{\Phi}$ be a $(0,3)$ Codazzi tensor on $(M, g)$.

(i) Let $\psi \in C^{\infty}(M)$ be the function s.t. $d \psi=T$ according to 3.3(ii), and assume that $\psi$ satisfies

$$
\int \psi e(2) \omega(g)=0 \quad \text { for any } \quad e(2) \in \mathfrak{C}_{2} .
$$

Then there exists $f \in C^{\infty}(M)$ such that $\bar{\Phi}$ can be generated by $f, \bar{\Phi}=\Phi(f)$.

(ii) If $f, f^{\#}$ generate $\bar{\Phi}$, then $f-f^{\#} \in \mathfrak{C}_{0}+\mathfrak{C}_{2}$.

Proof. We use the notation from (3.1.1) and consider the linear third order PDE-system

$$
\Phi(f)=\bar{\Phi}
$$

Taking the trace, we get

$$
\nabla_{k}(\triangle f+2(n+1) k f)=n T_{k}=n \partial_{k} \psi
$$

From the Fredholm alternative for elliptic operators [Nar68], the equation

$$
\triangle f+2(n+1) k f=n \psi
$$

has a solution if (3.4.1) is satisfied. Let $f$ be a solution of (3.4.4). We prove that $f$ also solves (3.4.2). Namely, $f$ generates a Codazzi tensor $\Phi(f)$, and both $(0,3)$-Codazzi tensors, $\Phi(f)$ and $\Phi$, have the same trace, thus $\Phi(f)=\bar{\Phi}$ from 2.5.

3.5. Corollary. Let $(M, g)$ as above and let the nonconstant function $f \in C^{\infty}(M)$ satisfy $\triangle f+$ $2(n+1) k f=0$. Then $f$ satisfies also $\Phi(f)=0$, i.e. $f \in \mathfrak{C}_{2}$. 


\section{Fourth order Codazzi tensors}

Gallot [Gal79] extended the investigations of Obata and Tanno to characterize Riemannian spheres by PDE-systems related to spherical harmonics. Similarly to Tanno's equation in section 3 we use Gallot's result on spherical harmonics of third order as motivation for the following.

4.1. Lemma and Definition. Let $(M, g)$ be a space of constant curvature $k$.

(i) For $f \in C^{\infty}(M)$, the $(0,4)$-tensor $\Psi(f)$ defined in local terms by

$$
\begin{aligned}
\Psi(f)_{i j k l}:= & \nabla_{l}\left\{\nabla_{k}\left[H(f)_{i j}\right]+k\left[f_{k} g_{i j}+f_{j} g_{i k}+f_{i} g_{j k}\right]\right\} \\
& +k\left[\nabla_{l} f_{k} g_{i j}+\nabla_{l} f_{j} g_{i k}+\nabla_{l} f_{i} g_{j k}\right]+k\left[\nabla_{j} f_{i} g_{k l}+\nabla_{k} f_{j} g_{i l}+\nabla_{k} f_{i} g_{j l}\right] \\
& +3 f k^{2}\left(g_{i j} g_{k l}+g_{j k} g_{i l}+g_{i k} g_{j l}\right) \\
= & \nabla_{l}\left\{\Phi(f)_{i j k}\right\}+k[\cdots]+3 f k^{2}(\cdots)
\end{aligned}
$$

is a $(0,4)$-Codazzi tensor. We say that $f$ generates the Codazzi tensor.

(ii) $f, f^{\#} \in C^{\infty}(M)$ generate the same Codazzi tensor $\Psi$ if and only if $\Psi\left(f-f^{\#}\right)=0$.

Proof. (i) The total symmetry of $\Psi(f)$ follows immediately from the fact that $\Phi(f)$ is a $(0,3)$ Codazzi tensor, thus $\nabla \Phi(f)$ is totally symmetric; the symmetry of the other terms is obvious.

(ii) The total symmetry of $\nabla \Psi(f)$ follows again from a lengthy, but straightforward calculation using the Ricci-identities ([Eis49], p. 30).

4.2. Notation. We introduce the following notation.

$$
\begin{aligned}
& \mathfrak{C}_{1}:=\left\{f \in C^{\infty}(M) \mid f \notin \mathfrak{C}_{0}, H(f)=0 \quad \text { on } M\right\} \cup\{0\}, \\
& \mathfrak{C}_{3}:=\left\{f \in C^{\infty}(M) \mid f \notin \mathfrak{C}_{1}, \Psi(f)=0 \text { on } M\right\} \cup\{0\} .
\end{aligned}
$$

The following properties are immediate consequences of 4.1.

4.3. Properties of $\Psi(f)$. Let $(M, g)$ be of constant curvature $k$.

(i) $\Psi(f)$ can be reformulated in terms of $H(f)$ by

$$
\begin{aligned}
\Psi(f)_{i j k l}= & \nabla_{l} \nabla_{k} H(f)_{i j}+2 k\left[H(f)_{k l} g_{i j}+H(f)_{j l} g_{i k}+H(f)_{i l} g_{j k}\right] \\
& +\left[H(f)_{i j} g_{k l}+H(f)_{j k} g_{i l}+H(f)_{i k} g_{j l}\right] ;
\end{aligned}
$$

in particular $f \in \mathfrak{C}_{1}$ implies $H(f)=0$, thus $\Psi(f)=0$;

(ii) For $f \in C^{\infty}(M)$, the symmetric $(0,2)$-tensor trace ${ }_{g}(\Psi)$ satisfies

$$
\operatorname{trace}_{g} \Psi=\operatorname{Hess} F+k g F,
$$

where $F:=\triangle f+3(n+2) k f$, and

$$
\operatorname{trace}_{g}^{2} \Psi=\triangle F+n k F=\triangle \triangle f+2(2 n+3) k \Delta f+3 n(n+2) k^{2} f .
$$

(iii) trace $_{g} \Psi(f)$ is a $(0,2)$-Codazzi tensor with generating function $F:=\triangle f+3(n+2) k f$;

(iv) trace $_{g} \Psi=0$ implies $F \in \mathfrak{C}_{1}$ for $k \neq 0$ and $F \in \mathfrak{C}_{0}$ for $k=0$. 
4.4. Theorem. Let $(M, g) \cong\left(S^{n}(k)\right.$, can). Let $\bar{\Psi}$ be a $(0,4)$-Codazzi tensor on $(M, g)$. Then

(i) there exists $F \in C^{\infty}(M)$ such that ([OS83], (2.3))

$$
\operatorname{trace}_{g} \bar{\Psi}=H(F)=\text { Hess } F+k g F .
$$

$F$ is unique modulo an additive first order spherical harmonic.

(ii) Let $F$ be a solution of (4.4.1) such that $F$ satisfies

$$
\int F e(3) \omega(g)=0 \quad \text { for all } \quad e(3) \in \mathfrak{C}_{3} ;
$$

then there exists $f \in C^{\infty}(M)$ which solves the PDE-system

$$
\Psi(f)=\bar{\Psi} .
$$

(iii) $f, f^{\#}$ satisfy $\Psi(f)=\Psi\left(f^{\#}\right)$ if and only if $f-f^{\#} \in \mathfrak{C}_{1}+\mathfrak{C}_{3}$.

Proof. Consider the PDE-system (4.4.3) for $\bar{\Psi}$ given and $f$ as unknown function. $\operatorname{trace}_{g} \bar{\Psi}$ is a $(0,2)$-Codazzi tensor. To solve (4.4.3) we first consider the PDE-system (4.4.1) for $F$. The system (4.4.1) has a solution $F$. We consider now the equation

$$
\triangle f+3(n+2) k f=F
$$

and apply the Fredholm alternative for elliptic operators ([Nar68], §3.9); (4.4.4) has a solution if the integrability condition (4.4.2) is satisfied. If (4.4.2) is satisfied for one solution $F$ of (4.4.1) then it is satisfied for any. Fix one solution $F$ of (4.4.1), let $f$ be a solution of (4.4.4) and consider the $(0,4)$-Codazzi tensor $\Psi(f)$ generated by $f$. Then

$$
\begin{aligned}
\operatorname{trace}_{g} \Psi(f) & =\operatorname{Hess}(\triangle f+3(n+2) k f)+k(\Delta f+3(n+2) k f) g \\
& =\operatorname{Hess} F+k F g=\operatorname{trace}_{g} \bar{\Psi} .
\end{aligned}
$$

Note that $\operatorname{Hess} F+k F g=\operatorname{Hess}(F+e(1))+k g(F+e(1))$ for $e(1) \in \mathfrak{C}_{1}$. Apply again 2.5. This gives $\Psi(f)=\bar{\Psi}$, i.e. $f$ solves (4.4.3).

4.5. Corollary. Let $(M, g)$ be as above and assume $f$ is nonconstant and satisfies

$$
\triangle f+3(n+2) k f=0 \text {. }
$$

Then $f \in \mathfrak{C}_{3}$.

4.6. Corollary. Let $(M, g)$ be as above and assume that $f \in C^{\infty}(M)$ satisfies

$$
\triangle \triangle f+2(2 n+3) k \Delta f+3 n(n+2) k^{2} f=0 .
$$

Then $f \in \mathfrak{C}_{1}+\mathfrak{C}_{3}$.

4.7. Observation. There is a different prooffor 4.6. To state the result consider two eigenvalues $\lambda, \mu$ of the Laplace operator with corresponding eigenspaces $E_{\lambda}, E_{\mu}$ on an arbitrary Riemannian manifold. Assume that $f \in C^{\infty}(M)$ satisfies the fourth order PDE

$$
\triangle \triangle f+(\lambda+\mu) \triangle f+\lambda \mu f=0 .
$$

(a) If $\lambda \neq \mu$, then $f \in E_{\lambda}+E_{\mu}$.

(b) If $M$ is closed and $\lambda=\mu$ then $f \in E_{\lambda}$. 
Proof. (a) $f$ satisfies the two equations

$$
\triangle(\triangle f+\lambda f)+\mu(\triangle f+\lambda f)=0 \quad \text { and } \quad \triangle(\triangle f+\mu f)+\lambda(\triangle f+\mu f)=0,
$$

thus $\triangle f+\lambda f=: f_{\mu} \in E_{\mu}, \triangle f+\mu f=: f_{\lambda} \in E_{\lambda}$ and $(\mu-\lambda) f=f_{\lambda}-f_{\mu}$.

(b) (4.7.1) implies $\triangle(\triangle f+\lambda f)+\lambda(\triangle f+\lambda f)=0$ thus $f_{\lambda}:=\Delta f+\lambda f \in E_{\lambda}$. Using Greens theorem and (4.7.1) we get:

$$
\begin{aligned}
\int f_{\lambda}^{2} \omega(g) & =\int\left\{(\triangle f)^{2}+2 \lambda f \triangle f+\lambda^{2} f^{2}\right\} \omega(g) \\
& =\int f\left\{\triangle \triangle f+2 \lambda \triangle f+\lambda^{2} f\right\} \omega(g)=0 .
\end{aligned}
$$

This gives $\triangle f+\lambda f=f_{\lambda}=0$.

\section{Characterization of Riemannian spheres}

Let $(M, g)$ be a Riemannian manifold and $f \in C^{\infty}(M)$ a nonconstant function. We extend the results of Obata, Tanno, etc. mentioned in section one.

5.1. Theorem. Let $\operatorname{dim}(M)=2$ and $M$ be closed of genus zero. Assume that there exists a positive constant $k$ and a nonconstant function $f$ generating a Codazzi tensor by one of the following assumptions (i), (ii), (iii):

(i) $f$ and $k$ define a traceless (0,2)-Codazzi tensor $H(f)$ as given in (1.0.1);

(ii) $f$ and $k$ define a traceless $(0,3)$-Codazzi tensor $\Phi(f)$ as given in (3.1.1);

(iii) $f$ and $k$ define a traceless $(0,4)$-Codazzi tensor $\Psi(f)$ as given in (4.1.1).

Then $(M, g)$ is a Riemannian sphere of constant curvature $k>0$.

Proof. (i) It follows from Theorem 2.3 that trace $H(f)=0$ implies $H(f)=0$. Now the assertion follows from Obata's theorem [Oba62].

(ii) Analogously the assumption (ii) gives $\Phi(f)=0$; we apply now Tanno's theorem.

(iii) In this case we apply Gallot's result on the characterization of Riemannian spheres by properties of the third order spherical harmonics.

Similarly Theorem 2.4 gives the following result:

5.2. Theorem. Let $\operatorname{dim} M>2$ and $M$ be closed. Assume that $(M, g)$ is conformally flat and Ricci pinched

$$
\text { Ric }>\frac{(m-1) n}{n+2 m-4} k g,
$$

where $m=2,3$ or 4 . Assume that there exists a positive constant $k$ and a function $f$ such that $f$ generates a Codazzi tensor like in one of the following assumptions (i), (ii), (iii):

(i) $m=2$ and $H(f)$, as given in (1.0.1), is a traceless (0,2)-Codazzi tensor;

(ii) $m=3$ and $\Phi(f)$, as given in (3.1.1), is a traceless (0,3)-Codazzi tensor;

(iii) $m=4$ and $\Psi(f)$, as given in (4.1.1), is a traceless (0,4)-Codazzi tensor.

Then $(M, g)$ is a Riemannian sphere of curvature $k$. 


\section{Codazzi fields, projective structures, differentiable spheres}

In this chapter we consider the behaviour of Codazzi fields under projective changes. We apply the results to the case of projectively flat differentiable spheres.

6.1. Definition. Let $\nabla$ be torsion free and denote by $\mathcal{P}=\mathcal{P}(\nabla)$ the class of all torsion free connections $\nabla^{\#}$ which are projectively related to $\nabla$. According to a result of Weyl, for $\nabla^{\#} \in \mathcal{P}$, there exists a one-form $\theta$ s.t.

$$
\nabla_{u}^{\#} v=\nabla_{u} v+\theta(u) v+\theta(v) u
$$

We call $0<\beta \in C^{\infty}(M)$ a projective factor if $\theta=d \ln \beta$ on $M$. We use the notation

$$
\nabla \stackrel{\beta}{\rightarrow} \nabla^{\#}
$$

for this particular projective change in $\mathcal{P}$.

6.2. Transformation Lemma. Let $\Phi$ be a symmetric $(0, m)$-tensor, let $\nabla \stackrel{\beta}{\rightarrow} \nabla^{\#}$ be a projective change. Then

(i)

$$
\begin{aligned}
& \left(\nabla_{v}^{\#}\left(\beta^{m-1} \Phi\right)\right)\left(u, w_{2}, \ldots, w_{m}\right)-\left(\nabla_{u}^{\#}\left(\beta^{m-1} \Phi\right)\right)\left(v, w_{2}, \ldots, w_{m}\right) \\
& =\beta^{m-1}\left\{\left(\nabla_{v} \Phi\right)\left(u, w_{2}, \ldots, w_{m}\right)-\left(\nabla_{u} \Phi\right)\left(v, w_{2}, \ldots, w_{m}\right)\right\}
\end{aligned}
$$

(ii) $\Phi$ is a $(0, m)$-Codazzi tensor relative to $\nabla$ if and only if the tensor $\beta^{m-1} \Phi$ is a $(0, m)$-Codazzi tensor relative to $\nabla^{\#}$.

Proof. (i) is a straightforward calculation, (ii) a consequence of (i). For $m=2$ see [PSS94].

Let $\nabla$ be a torsion free connection with symmetric Ricci tensor on $M \cong S^{n}$. Assume that $\nabla$ is projectively flat. It follows from [PSS94] $\S 1.7$, that $\nabla$ is projectively equivalent to a metric connection $\nabla^{\#}$, i.e. there exists a 1 -form $\theta$ on $M$ s.t.

$$
\nabla_{v} w-\nabla_{v}^{\#} w=\theta(v) w+\theta(w) v
$$

and $\nabla^{\#}=\nabla\left(g^{\#}\right)$.

As $\nabla^{\#}$ is projectively flat the metric must be of constant curvature $k$ and as $M \cong S^{n}$ we have $k>0$. Moreover, as $\nabla, \nabla^{\#}$ both are Ricci-symmetric, there exists (modulo constant positive factors) unique, oriented volume forms $\omega, \omega^{\#}$ s.t. $\nabla \omega=0, \nabla^{\#} \omega^{\#}=0$ ( $\omega^{\#}$ is the Riemannian volume form of $\left.g^{\#}\right)$. From this we get that $\theta=d \ln \beta$ for some positive function $\beta \in C^{\infty}(M)$ as a projective factor for the projective change (6.2.1).

The following theorem is an immediate consequence of the result 6.2 and sections 3 and 4 , resp.

6.3. Theorem. Let $M \cong S^{n}$ be equipped with a projectively flat, Ricci-symmetric connection $\nabla$, let $\Phi$ be a $\nabla$-Codazzi tensor of order $m$. Let $\beta$ be the projective factor from 6.1 describing the projective change to a constant curvature connection $\nabla^{\#}$. Then 
(i) $\beta^{m-1} \Phi=: \Phi^{\#}$ is a $\nabla^{\#-C o d a z z i ~ t e n s o r ; ~}$

(ii) for $m=3, \Phi^{\#}$ can be generated by a function as in 3.1;

(iii) for $m=4$, $\Phi^{\#}$ can be generated by a function as in 4.1 .

6.4. Remark. Theorem 6.3 and (3.3.1) in [PSS94] suggest the following: If $f$ generates $\Phi^{\#}$, then $\left(\beta^{1-m} f\right)$ generates $\Phi$.

\section{Basics from relative hypersurface theory}

We apply foregoing results to nondegenerate hypersurfaces in Euclidean and affine differential geometry. For the convenience of the reader we recall the following facts and notations for hypersurfaces with relative normalization from [SSV91].

It is one of the well-known advantages of the relative concept that one can specialize to standard geometries like the equiaffine geometry of Blaschke, to centroaffine geometry and to Euclidean geometry. See chapter 4-6 in [SSV91] for details.

\section{Relative normalizations}

Let $A$ be a real affine space which is modeled on a vector space $V$ of dimension $n+1$ with $n \geq 2$. Let $V^{*}$ denote the dual space. Let $<,>: V^{*} \times V \rightarrow \mathbb{R}$ be the canonical pairing between $V^{*}$ and $V$. Denote by $\bar{\nabla}$ the flat affine connection on $A$. Consider a smooth hypersurface immersion of a differentiable, connected and oriented manifold $M$

$$
x: M \rightarrow A \text {. }
$$

For $p \in M$, we may identify the tangent space of $A$ and its dual with $V$ and $V^{*}$, resp.:

$$
T_{x(p)} A=V, \quad T_{x(p)}^{*} A=V^{*} .
$$

By $\{Y, y\}$ we denote a relative normalization of $x$; that means $Y$ is a (nowhere vanishing) conormal field, $y$ is transverse to $x$, and $\{Y, y\}$ satisfy

$$
<Y, d x(v)>=0, \quad<Y, y>=1, \quad<Y, d y(v)>=0 .
$$

A triple $\{x, Y, y\}$ denotes a non-degenerate hypersurface $x$ together with its relative normalization $\{Y, y\}$. That $x$ is a non-degenerate hypersurface means that $\operatorname{rank}(Y, d Y)=n+1$ (which is then true for any conormal field). The normalization $\{Y, y\}$ defines the relative conormal Gauß map $Y$ and the relative Gauß map (or relative spherical indicatrix) $y$

$$
Y: M \rightarrow V^{*}, \quad y: M \rightarrow V .
$$

As a consequence of the non-degeneracy of $x, Y$ is an immersion with transverse position vector $Y$, while $y$ need not be an immersion.

\section{Structure equations}

The relative structure equations for a given triple $\{x, Y, y\}$ read

$$
\begin{aligned}
\bar{\nabla}_{v} y & =d y(v)=-d x(S v) \\
\bar{\nabla}_{v} d x(w) & =d x\left(\nabla_{v} w\right)+h(v, w) y \\
\bar{\nabla}_{v} d Y(w) & =d Y\left(\nabla_{v}^{*} w\right)+\widehat{S}(v, w)(-Y)
\end{aligned}
$$

they contain the fundamental geometric quantities of relative hypersurface geometry. 


\section{Fundamental quantities}

For a given triple $\{x, Y, y\}$ we have:

(i) $h$ is a semi-Riemannian metric, called the relative metric; $\widehat{S}$ is a symmetric $(0,2)$-tensor field, called the relative Weingarten form;

(ii) $S$ is the relative Weingarten operator satisfying $\widehat{S}(v, w)=h(S v, w)$; thus $S$ is $h$-selfadjoint;

(iii) $\nabla, \nabla^{*}$ are torsion free connections which are conjugate relative to $h$; as a consequence, $h$ is a Codazzi tensor relative to $\nabla$ and $\nabla^{*}$; moreover the relative conormal connection $\nabla^{*}$ is projectively flat;

(iv) the difference tensor

$$
C:=\frac{1}{2}\left(\nabla-\nabla^{*}\right)
$$

and the Levi-Civita connection $\nabla(h)$ of $h$ satisfy

$$
\nabla=\nabla(h)+C, \quad \nabla^{*}=\nabla(h)-C,
$$

and the relative cubic form $\widehat{C}(u, v, w):=h(u, C(v, w))$ is symmetric;

(v) $C$ defines the relative Tchebychev form

$$
n \widehat{T}(v):=\operatorname{trace}\{w \mapsto C(v, w)\}
$$

the relative Tchebychev vector field $T$ is implicitly defined by $h(T, v)=\widehat{T}(v)$.

\section{The traceless tensor $\widetilde{C}$}

Relative geometry includes the study of invariants of an affine hypersurface which do not depend on a particular relative normalization. One of these invariants which historically was one of the first of this type is a symmetric $(1,2)$-tensor field which can be expressed in terms of any relative geometry of a given hypersurface.

7.1. Lemma. For a given non-degenerate hypersurface $x$ with relative normalization $\{Y, y\}$, define the traceless $(1,2)$-tensor field $\widetilde{C}$ by

$$
\widetilde{C}(v, w):=C(v, w)-\frac{n}{n+2}\{\widehat{T}(v) w+\widehat{T}(w) v+h(v, w) T\} .
$$

Then

(i) $\widetilde{C}$ is independent of the choice of the relative normalization;

(ii) $\widetilde{C} \equiv 0$ characterizes quadrics. 


\section{The relative support function}

We recall the definition of the relative support function for $\{x, Y, y\}$ with respect to a fixed point $x_{0} \in A$ :

$$
\rho:=\rho\left(x_{0}\right):=<Y, x_{0}-x>.
$$

7.2. Lemma. Denote by Hess $^{*} \rho$ the covariant Hessian of $\rho$ in terms of the conormal connection $\nabla^{*}$ and by Ric* the Ricci tensor of $\nabla^{*}$. Then $(n-1) \widehat{S}=$ Ric $^{*}$ and

$$
\operatorname{Hess}^{*} \rho+\frac{1}{n-1} \operatorname{Ric}^{*} \rho=h \text {. }
$$

7.3. Corollary. $h$ is a $(0,2)$-Codazzi tensor relative to $\nabla^{*}$.

Proof. Define, in analogy to (1.0.1),

$$
H^{*}(\rho):=\operatorname{Hess}^{*} \rho+\frac{1}{n-1} \operatorname{Ric}^{*} \cdot \rho ;
$$

as $\nabla^{*}$ is projectively flat a well known straightforward calculation, using the integrability conditions, gives that $H^{*}(\rho)=h$ is a $\nabla^{*}$-Codazzi tensor.

\section{The Euclidean normalization as relative normalization}

We consider the affine space $A$ equipped with an Euclidean structure; we denote the Euclidean space by $E$. A hypersurface $x: M \rightarrow E$ is non-degenerate if and only if the Gauß map $\mu: M \rightarrow V$ is a hypersurface immersion itself. Then the Euclidean third fundamental form $\mathrm{III}=: g^{*}$ is positive definite on $M$, thus a Riemannian metric with Levi-Civita connection $\nabla^{*}$. The second fundamental form II coincides with the relative metric $h(E)$ of this normalization:

$$
h(E)=\mathbb{I} .
$$

Here the mark " $E$ " indicates that the relative quantity $h$ is considered in terms of a Euclidean normalization. We use this mark in an obvious way.

7.4. Lemma. In terms of a Euclidean normalization we have:

(i) $\widehat{S}(E)=$ III $=g^{*}$;

(ii) $2 \widehat{C}(E)=\nabla^{*} \mathbb{I} \quad\left(\nabla^{*}\right.$-covariant derivative of $\left.\mathbb{I}\right)$;

(iii) $2 n \widehat{T}(E)=-d \ln \left|H_{n}(E)\right|$ where $H_{n}(E)$ is the Euclidean Gauß-Kronecker curvature;

(iv) the Euclidean support function $\rho(E)$ and the equiaffine support function $\rho(e)$ satisfy

$$
\rho(e)\left|H_{n}(E)\right|^{\frac{1}{n+2}}=\rho(E) ;
$$

(v) denote by $\widetilde{C}(E)_{i j k}:=\widetilde{C}_{i j}^{r} \Pi_{r k}$, then

$$
\begin{aligned}
\widetilde{C}(E) & =\widehat{C}(E)-\frac{1}{n+2} \operatorname{sym}(n \widehat{T}(E) \otimes \mathbb{I}) \\
& =\widehat{C}(E)+\frac{1}{2(n+2)} \operatorname{sym}\left(d \ln \left|H_{n}(E)\right| \otimes \mathbb{I}\right) .
\end{aligned}
$$




\section{PDEs for the Euclidean support function}

From 7.2 and (7.3.1) we get the well-known second order PDE for the support function $\rho(E)$ in terms of the third fundamental form metric $g^{*}=$ III which is a metric of constant curvature one:

$$
\operatorname{Hess}^{*} \rho(E)+\rho(E) g^{*}=\mathbb{I} .
$$

This equation already was stated in the introduction (1.0.3). From this equation we derive the following nonlinear third order system for $\rho(E)$ :

8.1. Theorem. Let $x: M \rightarrow E$ be a non-degenerate hypersurface. Then the symmetric $(0,3)$ tensor $\Phi(f)$ from 3.1 for $f=\rho(E)^{2}$ satisfies, in terms of the third fundamental form metric, the system

$$
\frac{1}{2} \Phi\left(\rho(E)^{2}\right)=2 \rho(E) \widetilde{C}(E)+\rho(E) \operatorname{sym}[d \ln \rho(e) \otimes \mathbb{I}]
$$

where $\operatorname{sym}[\cdots]$ denotes the total symmetrization of the $(0,3)$-tensor in brackets $[\cdots]$ and $\rho(e)$ is the equiaffine support function.

Proof. Using (8.0.1) and 7.4 (i), (iii) we get

$$
\begin{aligned}
\nabla_{k}^{*} \nabla_{j}^{*} \rho(E)_{i}^{2} & =2 \nabla_{k}^{*}\left(\rho(E)_{i} \rho(E)_{j}+\rho(E) \nabla_{j}^{*} \rho(E)_{i}\right) \\
& =2\left\{\rho(E) \nabla_{k}^{*} \nabla_{j}^{*} \rho(E)_{i}+\rho(E)_{k} \nabla_{j}^{*} \rho(E)_{i}+\rho(E)_{j} \nabla_{k}^{*} \rho(E)_{i}+\rho(E)_{i} \nabla_{k}^{*} \rho(E)_{j}\right\} \\
& =2\left\{\rho(E) \nabla_{k}^{*} \Pi_{i j}-\rho(E) \nabla_{k}^{*} \rho(E) g_{i j}^{*}+\operatorname{sym}\left[d \rho(E) \otimes \operatorname{Hess}^{*} \rho(E)\right]_{k i j}\right\}
\end{aligned}
$$

Using $7.4(\mathrm{v})$ and (ii) we get

$$
\begin{aligned}
\nabla^{*} \operatorname{Hess}^{*} \rho(E)^{2}= & 2 \rho(E)\{2 \widetilde{C}(E)+\operatorname{sym}[(d \ln \rho(e)-d \ln \rho(E)) \otimes \mathbb{I}]\} \\
& -d \rho(E)^{2} \otimes g^{*}+2 \operatorname{sym}\left[d \rho(E) \otimes \operatorname{Hess}^{*} \rho(E)\right] .
\end{aligned}
$$

Hence with (3.1.1)

$$
\begin{aligned}
\frac{1}{2} \Phi\left(\rho(E)^{2}\right)= & \frac{1}{2} \nabla^{*}\left(\operatorname{Hess}^{*} \rho(E)^{2}+\rho(E)^{2} \otimes g^{*}\right)+\frac{1}{2} \operatorname{sym}\left[d \rho(E)^{2} \otimes g^{*}\right] \\
= & \rho(E)[2 \widetilde{C}(E)+\operatorname{sym}\{d \ln \rho(e) \otimes \Pi\}] \\
& +\operatorname{sym}\{d \rho(E) \otimes \underbrace{\left(\operatorname{Hess}^{*} \rho(E)+\rho(E) g^{*}-\Pi\right)}_{=0}\}
\end{aligned}
$$

8.2. Corollary. Let $x: M \rightarrow E$ be a non-degenerate hypersurface. Then:

(i) $x$ is a proper affine sphere with center in the origin $x_{0}=0 \in E$ if and only if $\rho(E)\left(x_{0}\right)=$ $\rho(E)$ satisfies the nonlinear third order PDE

$$
\Phi\left(\rho(E)^{2}\right)=4 \rho(E) \widetilde{C}(E) .
$$

(ii) The tensor $\rho(E) \widetilde{C}(E)$ is a Codazzi tensor of third order relative to $\nabla^{*}$ if $x$ is a proper affine sphere. 
Proof. It is well known that the equiaffine support function $\rho(e)=\rho(e)\left(x_{0}\right)$ is constant if and only if $x$ is a proper affine sphere with center $x_{0}$. Thus (8.1.1) implies (8.2.1) on proper affine spheres. Vice versa, (8.2.1) and (8.1.1) together imply

$$
\operatorname{sym}[d \ln \rho(e) \otimes \Pi]=0,
$$

as $\rho(E) \neq 0$ almost everywhere on non-degenerate hypersurfaces. As $\operatorname{rank}(\mathrm{II})=n,(8.2 .2)$ implies

$$
0=\operatorname{trace}_{\mathbb{I}}[d \ln \rho(e) \otimes \mathbb{I}]=(n+2) d \ln \rho(e) .
$$

This proves (i). The second part (ii) follows from (i) and 3.1.

8.3. Corollary. Let $x: M \rightarrow E$ be a non-degenerate hypersurface. Then we have the equivalence of (i) and (ii):

(i) $f$ is a quadric with center $x_{0}=0 \in E$.

(ii) $\rho(E)=\rho(E)\left(x_{0}\right)$ satisfies the third order system

$$
\Phi\left(\rho(E)^{2}\right) \equiv 0
$$

Proof. Let $x$ be a quadric with center $x_{0}$. Then $\widetilde{C}(E)=0$ (see [SSV91], (7.1.1)) and $\rho(e)=$ const, as quadrics with center are proper affine spheres. Thus (8.1.1) gives (8.3.1). Vice versa, (8.3.1) and (8.1.1) imply

$$
2 \rho(E) \widetilde{C}(E)+\rho(E) \operatorname{sym}[d \ln \rho(e) \otimes \mathbb{I}]=0 .
$$

As trace ${ }_{\mathbb{I}} \widetilde{C}(E)=0$ (apolarity of $\widetilde{C}$ ) we get $\rho(e)=$ const. But then (8.3.2) implies $\widetilde{C}(E) \equiv 0$ in analogy to the argument in the foregoing proof.

8.4. Remark. It is well known that Tzitzeica [Tzi07], [Tzi08] used the relation 7.4 (iv) in dimension $n=2$ for the first study of proper affine spheres. Like curvature relations for Weingarten surfaces relations between curvature functions and support functions are of particular interest. In Euclidean geometry, monotone relations of the type

$$
H_{1}=F(\rho) \quad \text { or } \quad H_{2}=F(\rho)
$$

characterize Euclidean spheres within the class of ovaloids if the derivative of the function $F$ satisfies

$$
F^{\prime}(\rho) \leq 0
$$

The example of the rotational ellipsoid shows that there are compact surfaces which are not Euclidean spheres and where

$$
H_{1}=f(\rho) \quad \text { or } \quad H_{2}=f(\rho)
$$


and $f^{\prime}>0$. Namely, on the rotational ellipsoid we have

$$
\begin{aligned}
& H_{1}=c_{1} \rho(E)+c_{2} \rho(E)^{3} \\
& H_{2}=\frac{1}{\rho(e)^{4}} \rho(E)^{4}=c_{3} \rho(E)^{4}
\end{aligned}
$$

and $0<c_{i} \in \mathbb{R}$ for $i=1,2,3$.

The example was pointed out by S.S. Chern in [Che45], while the first global characterizations by relations of type (8.4.1), (8.4.2) are due to K.P. Grotemeyer [Gro53]; for analogous results see [Sim67], [Sim68] and [Lei95]. As a consequence from 8.3 we get the following corollaries.

8.5. Corollary. Let $x: M \rightarrow E$ be a centered hyperquadric. Then we have the following relation between Euclidean curvature and support functions, which in standard local terminology reads:

$$
\partial_{k}\left(\rho(E) \frac{H_{n-1}(E)}{H_{n}(E)}\right)=-g^{*(i j)} \mathbb{I}_{j k} \partial_{i} \rho(E) .
$$

Here $g^{*(i j)}$ are the components of the inverse matrix

$$
g^{*(i j)} g_{j k}^{*}=\delta_{k}^{i}
$$

If $n=2$ and $p \in M$ is not umbilic, we can introduce a local parametrization around py curvature lines. Then $(8.5 .1)$ reads

$$
\begin{aligned}
& \partial_{1}\left(R_{1}+R_{2}\right)=-\left(R_{1}+3 R_{2}\right) \partial_{1} \ln \rho(E), \\
& \partial_{2}\left(R_{1}+R_{2}\right)=-\left(3 R_{1}+R_{2}\right) \partial_{2} \ln \rho(E),
\end{aligned}
$$

where $R_{1}, R_{2}$ are inverse to the principal curvatures.

Proof. We have $\widetilde{C}(E) \equiv 0$ on a quadric. Insert (7.4 ii and v) and trace $g^{*} \mathbb{I}=n \frac{H_{n-1}(E)}{H_{n}(E)}$ into the equation $\operatorname{trace}_{g^{*}} \widetilde{C}(E) \equiv 0$.

We use the relations from (8.5.3) to characterize ellipsoids.

8.6. Corollary. Let $x: M \rightarrow E_{3}$ be an analytic ovaloid. Assume that, in terms of the parametrization in (8.5.2), the two relations in (8.5.3) are satisfied. Then $x$ is an ellipsoid.

Proof. Assume that $x$ is not a sphere. Then the set of umbilics consists of isolated points. Locally we can introduce the parametrization by curvature lines. The relations in (8.5.3) imply

$$
\operatorname{trace}_{g^{*}}\{4 \rho(E) \widetilde{C}+\rho(E) \operatorname{sym}[d \ln \rho(e) \otimes \mathbb{I}]\}=0
$$

on $M$. But $\left\{\nabla^{*}, \Phi\left(\rho(E)^{2}\right)\right\}$ define a Codazzi pair. From the trace condition and 3.4 we see that $\rho(E)^{2} \in \mathfrak{C}_{0}+\mathfrak{C}_{2}$, thus

$$
\Phi\left(\rho(E)^{2}\right) \equiv 0 .
$$

From $8.3 x$ is a quadric, and as $M$ is compact $x$ is an ellipsoid. 
8.7. Theorem. Let $x$ be a hyperovaloid. Then (i) and (ii) are equivalent:

(i) $x$ is an hyperellipsoid;

(ii) the function $F:=\rho(E)^{2}-c^{*}$ is a second eigenfunction for $\left(M, g^{*}\right)$, where the positive constant $c^{*}$ is given by

$$
c^{*}=\frac{\int_{M} \rho(E)^{2} \omega^{*}}{\int_{M} \omega^{*}} .
$$

8.8. Remark. Consider an hyperellipsoid in Euclidean space $\mathbb{R}^{n+1}$ given by the equation

$$
\sum_{i=1}^{n+1}\left(\frac{x^{i}}{a_{i}}\right)^{2}=1 .
$$

Then $c^{*}$ satisfies the relation

$$
c^{*}=\frac{1}{n+1} \sum_{i=1}^{n+1} a_{i}^{2} .
$$

Proof (of Theorem 8.7). Let $F:=\rho(E)^{2}-c^{*}$ satisfy the equation of a second eigenfunction in terms of the metric $g^{*}$ :

$$
\triangle^{*} F+2(n+1) F=0 .
$$

$F$ generates a Codazzi tensor $\Phi(F)$ according to 3.1 and trace $g_{g^{*}} \Phi(F)=0$ from (8.8.2). Corollary 3.5 implies $0=\Phi(F)=\Phi\left(\rho(E)^{2}\right)$, and from $8.3 x$ is a quadric. As $M$ is compact, $x$ is a hyperellipsoid. The converse is obvious from 8.3.

Proof (of Remark 8.8). Let $x$ be an hyperellipsoid given by (8.8.1). Define a linear mapping $L: V \rightarrow V$ by $L:=\left(\delta_{i j} a_{i}^{-2}\right)$ with respect to the standard basis, then $\langle L x, x\rangle=1$. Since $<L x, x_{i}>=0$ we conclude $\mu=\|L x\|^{-1} L x$.

Because $\rho(E)^{2}-c^{*}$ is a second order spherical harmonic there exists a traceless linear mapping $B$ such that $\rho(E)^{2}-c^{*}=\langle\mu, B \mu\rangle$ (see [Led95]). Using $\langle L x, x\rangle=1$ we get

$$
c^{*}=\rho(E)^{2}-<\mu, B \mu>=<\mu, x>^{2}-<\mu, B \mu>=\|L x\|^{-2}-<\mu, B \mu>.
$$

If $x$ is an eigendirection of $L$ we have $x^{i}=a_{i}$. Summation of (8.8.3) over all eigendirections of $L$ gives ( $B$ is traceless): $(n+1) c^{*}=\sum_{i} a_{i}^{2}$.

We now present some result from [Led95] adapted to our problem.

8.9. Definition and Lemma. Let $x: M \rightarrow A$ be a non-degenerate hypersurface with relative normalization $\{Y, y\}$. Define for $f \in C^{\infty}(M)$

$$
\Phi_{Y}(f)_{i j k}:=\nabla_{i}^{*} \nabla_{j}^{*} f_{k}+\frac{1}{n-1}\left[2 f_{i} \operatorname{Ric}_{j k}^{*}+f_{k} \operatorname{Ric}_{i j}^{*}+f_{j} \operatorname{Ric}_{i k}^{*}+2 f \nabla_{i}^{*} \operatorname{Ric}_{j k}^{*}\right],
$$

then $\Phi_{Y}(f)$ is a Codazzi tensor with respect to $\nabla^{*}$. Note that $\Phi_{\mu}(f)=\Phi(f)$ from (3.1.1).

8.10. Lemma. Let $x: M \rightarrow A$ be a non-degenerate hypersurface with relative normalizations $\{Y, y\}$ and $\left\{Y^{\#}, y^{\#}\right\}$. Let $\beta \in C^{\infty}(M)$ such that $Y^{\#}=\beta Y$; we have

$$
\Phi_{Y \#}\left(\beta^{2} f\right)=\beta^{2} \Phi_{Y}(f)
$$

for all $f \in C^{\infty}(M)$. 
Now we prove Theorem A from section 1.

Proof (of Theorem A). Define $\beta=H_{n}^{-\frac{1}{n+2}}$. If $H_{n}^{\frac{2}{n+2}}-c=\beta^{-2}-c$ is a second order spherical harmonic then $\Phi\left(\beta^{-2}\right)=0$. Now consider the conformal change $h(e)=\beta$ II. From Lemma 8.10 for cubic forms we get

$$
\Phi_{Y(e)}\left(\beta^{2} f\right)=\beta^{2} \Phi(f) \quad\left(f \in C^{\infty}(M)\right)
$$

Hence, for $f=\beta^{-2}$

$$
\Phi_{Y(e)}(1)=\beta^{2} \Phi\left(\beta^{-2}\right) .
$$

Since $\beta>0$, from the foregoing and 8.9 we have $0=\Phi_{Y(e)}(1)=\frac{2}{n-1} \nabla^{*}(e) \operatorname{Ric}^{*}(e)=2 \nabla^{*}(e) \widehat{S}(e)$. From [Wie97], Cor. 2.11, we now conclude that $x$ is an hyperellipsoid.

If $x$ is an hyperellipsoid then $\rho(E)^{2}-c^{*}$ with $c^{*}$ given in 8.8 is a second order spherical harmonic and $\rho(e)$ is constant. With 7.4 (iv) we conclude that $H_{n}^{\frac{2}{n+2}}-\frac{c^{*}}{\rho(e)^{2}}$ is a second order spherical harmonic, too. From $\rho(e)=\left(a_{1} \cdots a_{n+1}\right)^{\frac{2}{n+2}}$ we get the expression for $c=\frac{c^{*}}{\rho(e)^{2}}$ in Theorem A.

\section{References}

[BGS97] Neda Bokan, Peter Gilkey, and Udo Simon, Geometry of differential operators on Weyl manifolds, Phil. Trans. R. Soc. London 453 (1997), 2527-2536.

[Che45] S. S. Chern, Some new characterizations of the Euclidean sphere, Duke Math. J. 12 (1945), 270290.

[CY86] S. Y. Chen and C. T. Yau, Complete affine hypersurfaces, Part I. The completeness of affine metrics, Commun. Pure and Appl. Math. 39 (1986), 839-866.

[Eis49] L. P. Eisenhart, Riemannian Geometry, Princeton University Press, 1949, Second printing.

[Fer91] D. Ferus, A remark on Codazzi tensors in constant curvature spaces, in Springer Lecture Notes 838 (1991), 257.

[Gal79] S. Gallot, Équations différentielles charactéristique de la sphère, Ann. Sci. École Norm. Sup. (4) 12 (1979), 235-267.

[GKS95] R. B. Gardner, M. Kriele, and U. Simon, Generalized spherical functions on projectively flat manifolds, Result. Math. 27 (1995), 41-50.

[Gro53] K. P. Grotemeyer, Eine kennzeichnende Eigenschaft der Kugel, Archiv. Math. 4 (1953), 230-233.

[KN69] S. Kobayashi and K. Nomizu, Foundations of Differential Geometry, vol. I, II, Interscience Publishers, 1962, 1969.

[Led95] J. Leder, Erzeugung kubischer Formen durch Funktionen, Diploma-thesis, TU-Berlin, 1995.

[Lei95] K. Leichtweiss, On a problem of W. J. Firey in connection with the characterization of spheres, Math. Pannonica 6 (1995), no. 1, 67-75. 

[LSW] H. L. Liu, U. Simon, and C. P. Wang, Higher order Codazzi tensors on conformally flat spaces, Beitr. Algebra Geom., to appear.

[LSW98] H. L. Liu, U. Simon, and C. P. Wang, Codazzi tensors and the topology of surfaces, Ann. Glob. Anal. Geom. 16 (1998), 1-14.

[Nar68] R. Narashimhan, Analysis on Real and Complex Manifolds, North Holland Amsterdam, 1968.

[Oba62] M. Obata, Certain conditions for a Riemannian manifold to be isometric with a sphere, J. Math. Soc. Japan 14 (1962), 333-340.

[OS83] V. I. Oliker and U. Simon, Codazzi tensors and equations of Monge-Ampère type on compact manifolds of constant sectional curvature, J. reine ang. Math. 342 (1983), 35-65.

[Pog78] A. V. Pogorelov, The multidimensional Minkowski problem, John Wiley \& Sons New York, 1978.

[PSS94] U. Pinkall, A. Schwenk-Schellschmidt, and U. Simon, Geometric methods for solving Codazzi and Monge-Ampère equations, Math. Ann. 298 (1994), 89-100.

[Sim67] U. Simon, Minkowskische Integralformeln und ihre Anwendungen in der Differentialgeometrie im Großen, Math. Annalen 173 (1967), 307-321.

[Sim68] U. Simon, Kennzeichnungen von Sphären, Math. Annalen 175 (1968), 81-88.

[Sim95] U. Simon, Transformation techniques for partial differential equations on projectively flat manifolds, Result. Math. 27 (1995), 160-187.

[SS62] P. A. Schirokow and A. P. Schirokow, Affine Differentialgeometrie, Teubner Leipzig, 1962.

[SSV91] U. Simon, A. Schwenk-Schellschmidt, and H. Viesel, Introduction to the Affine Differential Geometry of Hypersurfaces, Science University of Tokyo, 1991, Lecture Notes [ISBN 37983 15299].

[Tan78] S. Tanno, Some differential equations on Riemannian manifolds, J. Math. Soc. Japan 30 (1978), 509-531.

[Tzi07] G. Tzitzeica, Sur une nouvelle classe de surfaces, Comptes Rend. Acad. Sci. Paris 145 (1907), 132-133.

[Tzi08] G. Tzitzeica, Sur une nouvelle classe de surfaces, Comptes Rend. Acad. Sci. Paris 146 (1908), 165-166.

[Wie97] M. Wiehe, Hypersurfaces with locally symmetric conormal connection, Preprint 546, Fachber. Math., TU Berlin, 1997, pp. 1-12.

\section{Addresses:}

J. Leder, U. Simon, M. Wiehe: FB Mathematik, TU Berlin, MA 8-3, Strasse des 17. Juni 135, D-10623 Berlin.

A. Schwenk-Schellschmidt: FB 2 Mathematik/Physik, TFH Berlin, Luxemburger Strasse 10, D-13353 Berlin.

e-mail:

judith@sfb288.math.tu-berlin.de

simon@sfb288.math.tu-berlin.de

wiehe@math.tu-berlin.de

schwenk@tfh-berlin.de 
